\title{
La diversificación geográfica en los destinos de exportación de las empresas y cooperativas agroalimentarias. Influencia de los factores externos para su selección
}

\section{Pilar Cos Sánchez, Blanca Escardíbul Ferrá, Antonio Colom Gorgues}

RESUMEN: La diversificación geográfica y la selección de mercados exteriores, aunque generalmente consideradas de forma independiente, conforman un amplio ámbito de estudio. La relación entre ambas disciplinas se ha visto obstaculizada por la multitud de enfoques de investigación en la selección de mercados y por su mayor estudio con otras decisiones estratégicas de internacionalización. A su vez, la importancia de la internacionalización para las cooperativas se hace evidente, identificándose como uno de los factores clave en su competitividad. Sin embargo, sus limitaciones internas y externas dificultan su expansión exterior. El propósito de este trabajo es determinar la influencia de los factores externos de selección de mercados exteriores en la tendencia a su diversificación, sobre una muestra de un tejido exportador concreto. Los datos de los países destino se recogen de diversas fuentes secundarias, tales como el Banco Mundial, la OCDE, la OMC, el World Economic Forum y el Hofstede Centre. Los resultados demuestran que el desarrollo económico y el tamaño del mercado se configuran como variables influyentes en la diversificación de destinos de exportación, no afectando, no obstante, la distancia psicológica. Las cooperativas analizadas, como parte de la economía estudiada, podrían valerse de los resultados obtenidos como referencia en el proceso de selección de mercados exteriores de destinos menos frecuentados, sobre todo por la tendencia de una mayor concentración de sus exportaciones a países de la UE.

PALABRAS CLAVE: Selección de mercados exteriores, diversificación geográfica, cooperativas agroalimentarias, internacionalización, factores del entorno, distancia psicológica.

CLAVES ECONLIT: F14, M16, C31.

Cómo citar este artículo/How to cite this article: COS, P., ESCARDÍBUL, B. \& COLOM, A. (2021): "La diversificación geográfica en los destinos de exportación de las empresas y cooperativas agroalimentarias. Influencia de los factores externos para su selección", CIRIEC-España, Revista de Economía Pública, Social y Cooperativa, 102, 161-195. DOI: 10.7203/CIRIEC-E.102.17840.

Correspondencia: Pilar Cos Sánchez, Universidad de Lleida, pilar.cos@udl.cat, ORCID: 0000-0001-7882-6847; Blanca Escardíbul Ferrá, Universidad de Lleida, blanca.escardibul@udl.cat, ORCID: 0000-0001-5165-3325; Antonio Colom Gorgues, Universidad de Lleida, antonio.colom@udl.cat, ORCID: 0000-0001-7129-2539. 


\section{Expanded abstract}

\section{Geographic diversification in export destinations of agri-food companies and cooperatives. Influence of external factors on their selection}

\section{Objectives}

The relationship of the disciplines object of this study, geographic diversification and international market selection (IMS), has been hampered by a high degree of fragmentation of the IMS in the various research streams, and by its greater link in favour of works focused on the entry mode into foreign markets. Although Tihanyi, Griffith and Russell (2005) study the effect of IMS factors on geographic diversification, their work focuses on cultural distance. In turn, in the work of Cos and Escardíbul (2019) is concluded that the diversification of export destinations of the companies is opening the way to use other criteria in the IMS that differ from geographic and cultural proximity. Although the research recognizes that IMS may be associated with the criteria most commonly used in the literature, it does not explicitly focus on its relationship with geographic diversification, suggesting, however, the influence of IMS factors.

The objective of this paper, focused on the geographic diversification of an economy, is to determine the impact of the most common factors of traditional internationalization theories to select countries. More specifically, it is intended to answer the following questions: a) Is psychological distance an influencing factor in the growing of geographic diversification of an economy? b) What is the relationship between the external factors of the IMS and a tendency to an economy towards greater geographic diversification?

This research focuses on a specific economy chosen for its particularities, the exporting agri-food sector of Lleida. From the perspective of exporting agri-food cooperatives, highlight the interest that the results obtained in this study may arouse. Although non-cooperative companies predominate over cooperatives in the exporting agri-food sector of this province, Lleida stands out as the most exporter of the Catalonia agri-food cooperative sector, concentrating more than half of the total exported and with two out of four exporting cooperatives (Cos, Aldaz, Escardíbul, Millán and Moltó, 2019). In turn, the horticultural is the main exporting sector of the Lleida agri-food cooperatives, as in the case with the total exports of this province.

\section{Methodology}

The study data are obtained from several secondary sources. The first one, from the Customs Department of the Spanish Tax Agency, is used to obtain the 23 countries that make up the 
sample, the main destinations of the 672 agri-food exporting companies in the province of Lleida. The importance that the agri-food cooperatives of this province acquire in these figures, still represented by $10 \%$, must be analysed in the context of the agri-food cooperative sector in Catalonia, as Lleida is the most internationalized province.

The growth of Lleida's agri-food exports, in the analysed period, has been considerably lower in the main destinations of the EU-28 than in the rest of the countries of both advanced and emerging economies, the latter acquiring greater prominence to the detriment of European Community destinations, despite its higher export quota. Regarding the Catalan cooperatives studied, they tend, on the one hand, to a greater diversification in the number of destinations, and, on the other hand, to a greater concentration of the percentage of exports to the EU-28 countries.

The dependent variable of this study, geographic diversification, when analysed from the general perspective of an economy and considering the dynamic nature of this strategy, is the 'Growth rate', during a certain period, of exports to each selected market. The independent variables used are psychological distance (cultural, geographic and language), economic intensity, country risk, a country's competitiveness and the market size. To measure these variables in each priority destination country, the information used comes from secondary sources: World Bank, World Development Indicators Database (WDI); OECD; WTO; Global Competitiveness Report (World Economic Forum); Hofstede Centre (2010); Geobytes Database; and Centre for the Practice of International Trade (Melbourne Business School).

Like other studies related to the scope of this research, a factor analysis is performed first (e.g., Whitelock and Jobber 2004; Sakarya et al., 2007; Sheng and Mullen 2011), followed by a multiple regression analysis (e.g., Triguero-Cano and Cuerva, 2011; Benito-Osorio et al., 2016) with the scores of the retained factors, avoiding the problem of correlation between the variables and the influence of many items.

The three working hypotheses are:

- H1. The psychological distance (cultural, geographic and language) positively influences the geographic diversification of an economy.

- H2a. Environmental factors (economic development) influence the geographic diversification of an economy.

- H2b. Environmental factors (market) influence the geographic diversification of an economy.

\section{Results / conclusions / limitations}

Four factors are obtained from the factor analysis ('Economic development', 'Market size,' 'Cultural / Language distance' and 'Geographic distance'), which together explain $88.1 \%$ of the variance. The results of the regression analysis allow the validation of $\mathrm{H} 2 \mathrm{a}$ and $\mathrm{H} 2 \mathrm{~b}$ hypotheses. The model shows a significant and negative relationship between economic development and the market size of an exporting country with the tendency of an economy to geographic diversification. In this way, companies tend to diversify their sales towards countries with less political and economic stability (lower GDP per capita, lower competitive intensity and with 
country risk) and with smaller market size. Regarding H1, with the results obtained, it could not be confirmed, as there was no empirical evidence of a relationship between both variables.

As part of the analysed economy, the agri-food cooperatives described in this paper could use these conclusions as a reference and comparison of the companies in the studied economy, considering their specific characteristics. The importance that international activity acquires in agri-food cooperatives for their growth (Bretos, Díaz-Foncea and Marcuello, 2018), makes this work of special interest for this group of companies, especially considering that the internal limitations and Cooperatives' externalities make them less competitive and hinder their international expansion. The influence of the relevant criteria of the IMS with various strategic internationalization decisions, such as geographic diversification, should serve the cooperatives in this study to be able to evaluate the different alternatives that are presented to them.

Regarding the main limitations, it is worth highlighting the exclusive use of secondary sources and the dependent variable used. Limitations, however, that do not significantly influence the results and that could open the way to future research.

\section{Original value and practical implications}

With the present work it has been tried to continue advancing in the knowledge of the internationalization of the Spanish company, among which is the cooperative sector, providing new empirical evidence on a little-discussed topic, such as the relationship between IMS and international diversification. Thus, an integrative analysis of the external factors of the IMS that influence the geographic diversification of a specific economy is provided, based on secondary sources that provide us with objective information and immediate accessibility.

From the present study, a series of practical implications can be found in the field studied. Diversifying destinations should help companies to become aware of the importance of selecting markets in their internationalization process, applying, for this, a methodology with a systematic approach or not, which allows them to expand their range of target markets. Above all, considering that companies in their territory and sector, whether they are competitors or not, seek new options to direct their international sales, beyond the security of community markets, geographically close and favoured by belonging to a common market. Probably these companies that target markets that are not desirable a priori due to their macroeconomic characteristics, select them for a series of reasons that, although unknown in this study, offer them some competitive advantage that makes them suitable.

For the agri-food cooperative sector, the usefulness of these practical implications acquires special relevance, when operating, most of the large global cooperatives of the agri-food sector, at an international level (Bretos et al., 2018), standing out, in turn, the export as entry mode compared to the rest of the modalities. With these premises, the cooperative sector studied still has a journey in its internationalization, since half of the agri-food cooperatives in Lleida still do not export. In addition, with a tendency towards the concentration of their exports to EU countries in terms of volume, they could be based on environmental factors to diversify their foreign target markets, increasing their exports in those markets already served, with still marginal shares. Thus, geographic proximity and EU membership are the criteria main- 
ly used by the cooperatives studied to select foreign markets, typical of the non-systematic approach, which implies that to choose export countries from other advanced or emerging economies they should develop systematic models, which would provide cooperatives with a structured method in the IMS, with the benefits that this entails.

Keywords: International market selection, geographic diversification, agri-food cooperatives, internationalization, environmental factors, psychological distance. 


\section{Introducción}

La elección de la estrategia de expansión de mercados exteriores es una de las decisiones clave que debe adoptar la empresa en su proceso de internacionalización, una vez ésta decide involucrarse en actividades de exportación (Cavusgil y Zou, 1994). Desde el punto de vista del marketing internacional, esta decisión, tomada a largo plazo, requiere: i) la identificación previa y análisis de los mercados potenciales, así como, establecer el orden de entrada en los mismos (Ayal y Zif, 1979); ii) decidir el grado temporal de expansión o ritmo de expansión internacional (Ayal y Zif, 1979); iii) la determinación del número de mercados exteriores en los que competirá u operará (Albaum, Strandskov y Duerr, 2002), teniendo en cuenta que dispone de un presupuesto de marketing limitado, que deberá ser repartido entre los diferentes mercados seleccionados (Ayal y Zif, 1979); iv) y por último, definir las características deseadas en estos mercados (Albaum et al., 2002).

De esta forma, la selección de mercados exteriores (SME) y la estrategia de expansión internacional seguida por la empresa, aunque generalmente consideradas de forma independiente, conforman un amplio ámbito de estudio. Se hace necesaria una selección previa para adoptar una estrategia de expansión internacional determinada, o a la inversa, el enfoque de expansión condicionará el número de mercados finalmente seleccionados y su naturaleza.

De las investigaciones sobre la internacionalización se desprende que, el paso de seleccionar el país de destino de las exportaciones o inversiones es una decisión que la empresa deberá tomar antes de decidir qué modo de entrada utilizar (Johanson y Vahlne, 1990). Es ésta última decisión el centro de atención de numerosos investigadores en el área de los negocios internacionales (Werner, 2002), a diferencia del menor número de trabajos enfocados en la decisión de seleccionar los países donde proyectar sus ventas exteriores (Andersen y Buvik, 2002). A su vez, Koch (2001) muestra como tendencias destacadas en la literatura de selección del modo de entrada y de nuevos mercados: el predominio del interés en el entorno externo sobre el papel del entorno interno de la empresa; el predominio del enfoque prescriptivo sobre el descriptivo; la perspectiva estática; y la focalización en los aspectos cuantitativos del proceso frente a los cualitativos.

Una de las primeras decisiones en la selección de mercados internacionales debería identificar los criterios relevantes a partir de los cuales se evaluarán las diferentes alternativas (Andersen y Buvik, 2002). Al realizar una revisión en profundidad de los trabajos publicados dentro esta temática, se advierte una amplia variedad de criterios, en los que se incluyen principalmente indicadores generales del país, tales como las características macroeconómicas, políticas y culturales, así como indicadores específicos del mercado, tales como el tamaño de este, la competencia, los canales de distribución y los costes de operar en los mercados. Los criterios identificados diferirán según la finalidad del estudio y el enfoque que se adopte, tal como queda reflejado en la extensa literatura en este ámbito de estudio y que se expone a lo largo del artículo.

En cuanto a la estrategia de expansión de mercados, se han identificado y discutido dos opciones estratégicas alternativas, concentración versus diversificación (e.g. Ayal y Zif, 1979; Lee 
y Yang, 1990; Mas, Nicolau y Ruiz, 2006; Losada, Ruzo, Barreiro y Navarro, 2007), definidas en función del número de mercados en los que la empresa trata de afirmar su presencia, es decir, centrarse en un número relativamente pequeño de mercados clave, para lograr una mayor cuota de mercado o entrar simultáneamente en un elevado número de mercados generalmente dispersos, con bajas participaciones. Si bien la diversificación geográfica o internacional ha recibido una gran atención en las últimas décadas en diversas disciplinas, tales como la internacionalización, el marketing, las finanzas y contabilidad (Hitt, Tihanyi, Miller, \& Connelly, 2006), casi toda la literatura está centrada en las multinacionales y en las IDE (inversiones directas en el exterior), y en su relación con el resultado de la empresa. Además, no existen prácticamente estudios empíricos que hayan examinado simultáneamente el comportamiento exportador en una muestra única de grandes empresas y pymes (Benito-Osorio, Colino, Guerras-Martín y Zúñiga-Vicente, 2016).

La diversificación internacional generalmente ha estado relacionada con un mejor resultado exportador (e.g. Lee y Yang 1990; Alonso y Donoso 1998; Navarro, 2002; Chao, Kim, Zhao y Hsu, 2012; Cos, Colom y Cabasés, 2019), aun no existiendo consenso al respecto, al encontrarse, en menor medida, enfoques que apuntan a la no existencia de asociación o a una relación negativa. Autores de este último enfoque sugieren que a medida que aumenta el número de países extranjeros atendidos, se podrían generar costos adicionales que superasen los beneficios incrementales de la empresa (Benito-Osorio et al., 2016). Asimismo, la literatura se ha centrado principalmente en el análisis de los factores de carácter interno ${ }^{1}$ de la empresa como determinantes de la diversificación geográfica (e.g. Ayal y Zif, 1979; Katsikea et al., 2005; Navarro, 2002; Mas et al., 2006; Losada et al., 2007; Boateng et al., 2008; Cos et al., 2019a).

La relación de las disciplinas objeto del presente estudio, diversificación geográfica y selección de mercados exteriores, se ha visto obstaculizado por un alto grado de fragmentación de ésta última en las diversas corrientes de investigación, y por su mayor vínculo a favor de trabajos enfocados hacia la decisión sobre las formas de entrada en mercados internacionales. Si bien Tihanyi, Griffith y Russell (2005) estudian el efecto de los factores de la SME en la diversificación geográfica, su trabajo se focaliza en la distancia cultural. A su vez, en el trabajo de Cos y Escardíbul (2019) se concluye que la diversificación de destinos de exportación de las empresas va abriendo paso al uso de otros criterios en la SME diferentes a la proximidad geográfica y cultural. Aunque la investigación reconoce que la SME puede estar asociada a los criterios más comúnmente utilizados en la literatura, ésta no se enfoca explícitamente a su relación con la diversificación geográfica, dejando intuir, sin embargo, la influencia de los factores de la SME.

La contribución del presente artículo radica en cubrir este espacio de investigación en el contexto de las empresas internacionales, entre las que se encuentran el sector cooperativo, ofreciendo un análisis conjunto de los diversos factores externos en la SME que, podrían afectar en la tendencia a la diversificación de destinos de una economía en particular. Así, en este trabajo se proporciona un análisis integrador en el proceso de representar la relación existen-

1. Sobre todo, características de la empresa (tamaño, sector, antigüedad, ...), factores del producto y del marketing. 
te entre diversificación geográfica-selección de mercados exteriores, a partir de una revisión previa de la literatura en ambos ámbitos de estudio.

El objetivo de este artículo, centrado en la diversificación geográfica de una economía, es determinar el impacto de los factores más obvios y usuales ${ }^{2}$ de las teorías tradicionales de internacionalización, para seleccionar los países. De forma más concreta, se pretende responder a las siguientes cuestiones: a) ¿Es la distancia psicológica ${ }^{3}$ un factor influyente en la creciente diversificación geográfica de una economía?; b) ¿Qué relación existe entre los factores externos de la selección de mercados exteriores y la tendencia de una economía a una mayor diversificación geográfica?

Para el contexto empírico del estudio se toma como referencia la evolución de los destinos prioritarios de exportación de una muestra de empresas españolas, cuya información procede del Departamento de Aduanas de la Agencia Tributaria de España (AEAT) ${ }^{4}$, partiendo del trabajo de Cos y Escardíbul (2019). Esta investigación se centra en una economía concreta elegida por sus particularidades, el sector agroalimentario exportador de Lleida, sector que representa casi el $70 \%$ del total exportado y se encuentra entre las diez primeras provincias españolas tanto por su mayor volumen de exportaciones agroalimentarias como por su mayor tasa de cobertura comercial. Asimismo, las exportaciones agroalimentarias de esta provincia han crecido a un ritmo superior a las del conjunto de Cataluña y España, con un incremento en el periodo analizado (2010-2014) del 46\%. En el estudio citado se constata una tendencia a la diversificación geográfica de los destinos de sus exportaciones durante los cinco años estudiados, con un crecimiento progresivo hacia áreas tanto de economías emergentes como de economías avanzadas menos frecuentadas. Este aumento de las exportaciones se produce principalmente en países de América Latina, de Asia (incluido Oriente Medio) y África, en detrimento de destinos tradicionales y más seguros para las empresas españolas, como son de la UE-28 y el resto de Europa, aun manteniendo una fuerte presencia en estos mercados más consolidados ${ }^{5}$.

En la medición de las variables, para cada uno de los países seleccionados, se emplean los datos disponibles de diversas fuentes de información secundarias, entre ellas el Banco Mundial, la OCDE (Organización para la Cooperación y Desarrollo Económicos), la OMC (Organización Mundial de Comercio), el World Economic Forum y el Hofstede Centre.

Desde la perspectiva de las cooperativas agroalimentarias exportadoras, destacar el interés que puede suscitar los resultados obtenidos en el presente estudio. Aun predominando las empresas no cooperativas sobre las cooperativas en el sector exportador agroalimentario de esta provincia, Lleida sobresale como la más exportadora del sector cooperativo agroalimentario de Cataluña, concentrando más de la mitad del total exportado y con dos de cada cuatro cooperativas exportadoras (Cos, Aldaz, Escardíbul, Millán y Moltó, 2019). A su vez, es el sector

\section{Distancia psicológica y geográfica; factores del entorno, del mercado y de la competencia.}

3. Véase explicación en apartado 2.1 de este artículo.

4. Consultado en la base de datos de DataComex, del Ministerio de Economía y Competitividad de España.

5. La UE-28 sigue siendo el principal destino de las exportaciones agroalimentarias de Lleida, ya que el 69\% de sus exportaciones están dirigidas a los diez primeros países destino, de los cuales seis son países comunitarios. 
hortofrutícola el principal exportador de las cooperativas agroalimentarias de Lleida, igual que ocurre con las exportaciones totales de esta provincia. De forma que, adicionalmente, se realiza una descripción del sector cooperativo mencionado, para situarlo en el contexto de la economía analizada.

Es relevante señalar que el cooperativismo agroalimentario exportador de España tiene un papel relevante en las exportaciones del sector agroalimentario español, con una cuota de exportación del 18\%, tal como se detalla en el informe de la organización 'Cooperativas Agro-alimentarias de España', del año 2017. Por tanto, se observa una creciente presencia de las cooperativas españolas en los mercados internacionales, siendo las cooperativas más dimensionadas las que cuentan con mayores tasas de exportación.

El trabajo se estructura del siguiente modo. Tras esta introducción, en el siguiente apartado se desarrolla el marco teórico de las dos disciplinas estudiadas y de su vínculo, para a continuación desarrollar las hipótesis de trabajo. En tercer lugar, se dedica un apartado a la literatura de la internacionalización de las cooperativas agroalimentarias, terminando con una descripción del sector del cooperativismo agroalimentario de Cataluña. Le sigue un cuarto apartado sobre la metodología utilizada y otro concerniente a los resultados obtenidos. Finalmente, se recogen las conclusiones, donde también se ofrecen unas implicaciones prácticas y algunas de las pautas para futuras investigaciones de esta temática.

\section{Factores externos de la SME condicionantes de la diversificación geográfica}

La selección de mercados exteriores es el primer paso en la proyección internacional de una empresa y una de las decisiones más determinantes dentro del proceso de internacionalización de la empresa (Papadopoulos y Martín, 2011). La importancia de elegir los mercados internacionales más adecuados para una empresa ha sido ampliamente reconocida, tanto por su relación con otras decisiones estratégicas (entre ellas formas de entrada, marketing internacional, resultado exportador y diversificación internacional) como por tratarse de una materia esencial en el marketing internacional y en la investigación, estrategia y gestión internacional de las empresas (Gastón-Breton y Martín, 2011).

En el trabajo de Ozturk, Joiner y Cavusgil (2015) se detalla de forma exhaustiva los criterios más usuales propuestos en la SME, entre los que se destacan aquellos concernientes al entorno, al mercado y a la distancia psicológica, así como de una recopilación de los principales estudios en esta materia. De acuerdo con la clasificación ofrecida por Papadopoulos y Denis

6. Es la Organización que representa y agrupa a las cooperativas, con un total de 1.182.346 socios en todo el territorio nacional, en prácticamente todos los subsectores agroalimentarios. 
(1988), éstos se dividen en modelos conceptuales (e.g. Papadopoulos y Martín, 2011), de agrupación (grouping studies) (e.g. Cavusgil, Kiyak, \& Yeniyurt, 2004) y de estimación (estimation studies) (e.g. Sakarya, Eckman, \& Hyllegard, 2007). Además de estos modelos de enfoque sistemático ${ }^{7}$, el enfoque no sistemático ${ }^{8}$ es ampliamente utilizado por las empresas, donde prima la proximidad geográfica (Dow 2000; 0jala y Tyrväinen 2008; Sheng y Mullen 2011) y cultural (Dow y Karunaratna, 2006) en la selección de mercados, y donde la decisión proactiva del enfoque sistemático se ve sustituida por una decisión reactiva del no sistemático. Los modelos sistemáticos pueden ser demasiado complejos para ser aplicables en la práctica (Papadopoulos et al., 2002), a diferencia de los modelos no sistemáticos que reducen la complejidad de evaluar diferentes mercados a través de una amplia búsqueda de información objetiva, fundamentándose en un factor influyente clave, la distancia psicológica, siguiendo el modelo de Uppsala (Johanson y Vahlne, 2009). No obstante, la selección de mercados internacionales sistemática es un factor determinante del resultado exportador (Brouthers y Nakos 2005; Brouthers et al., 2009), al contrario de los no sistemáticos que, aunque prácticos, pueden no obtener los resultados deseables. Por otro lado, He, Lin y Wei (2016) sugieren que, integrando ambos enfoques, las empresas pueden conseguir mejores resultados en sus decisiones de expansión global.

A partir de la exposición de los modelos y criterios en la SME, distintas investigaciones previas estudian su interrelación con diferentes decisiones estratégicas en la internacionalización, como son la forma de entrada, la estrategia de marketing, el grado de internacionalización y la diversificación internacional, así como aquellos trabajos vinculados al resultado exportador (Tabla 1).

\section{Tabla 1. Relación factores de SME con internacionalización}

\begin{tabular}{l|l} 
Variable de internacionalización & Estudio \\
\hline Diversificación internacional & Tihanyi et al., 2005 \\
\hline Formas de entrada & $\begin{array}{l}\text { Shama, 2000; Koch, 2001; Tihanyi et al., 2005; Rothaermel } \\
\text { et al., 2006; Quer, Claver y Rienda, 2007; Brouthers y } \\
\text { Hennart, 2007; Ojala y Tyrväinen 2008; Dow y Larimo, 2011; } \\
\text { Rienda, Claver y Quer, 2013; Quer, Claver y Rienda, 2017. }\end{array}$ \\
\hline Estrategia de marketing & $\begin{array}{l}\text { Papadopoulos y Denis, 1988; Powers y Loyka, 2010; Sousa y } \\
\text { Lages, 2011; Navarro-García et al., 2014. }\end{array}$ \\
\hline Grado de internacionalización & Javalgi y Grossman, 2014. \\
\hline Resultado exportador & $\begin{array}{l}\text { Brouthers y Nakos, 2005; Brouthers et al., 2009; Navarro- } \\
\text { García et al., 2014. }\end{array}$ \\
\hline
\end{tabular}

Fuente: Elaboración propia.

7. El proceso de toma de decisiones está estructurado y formalizado, de tal forma que el decisor tendrá que pasar por una serie de etapas para llegar a tomar la decisión correcta (Andersen y Buvik 2002).

8. No se utiliza ningún método en el proceso de selección y tiene una naturaleza descriptiva para determinar cómo se comportan las empresas a la hora de seleccionar mercados exteriores. 
A continuación, se exponen los resultados obtenidos en la relación entre los factores de la SME y las medidas habitualmente utilizadas en la literatura de la internacionalización (Tabla 1), a falta de estudios que los asocien con la diversificación geográfica y teniendo en cuenta el carácter multidimensional de la internacionalización.

\subsection{Distancia psicológica}

El concepto de distancia psicológica (DP) es esencial para valorar las diferencias entre países y para gestionar la SME. A pesar de las diversas definiciones, clasificadas por Sousa y Lages (2011) en tres categorías, en la revisión de la literatura de la internacionalización encontramos un mayor número de estudios que utilizan únicamente una medida, principalmente la distancia cultural o geográfica. De esta forma, en la SME, la distancia geográfica y cultural son identificados como criterios de gran relevancia (e.g. He y Wei, 2011; Sakarya et al., 2007). Según Kogut y Singh (1988) y Hofstede (1989), la distancia cultural nos indica la diferencia en cuanto a la cultura del país de origen y cada uno de los países de destino de exportación o de inversión.

La diversidad lingüística, aunque menos utilizada en la literatura de SME, motivado probablemente por su complejidad y su falta de acuerdo en las medidas a utilizar (Dow y Karunaratna 2006), es un factor a considerar en la distancia psicológica. Sheng y Mullen (2011), en su estudio, que clasifican los mercados más atractivos para empresas de EEUU a través de un modelo híbrido, encuentran que la mayor diversidad lingüística entre dos países condiciona negativamente su selección, de la misma forma que para Dow y Karunaratna (2006) condiciona el comercio bilateral.

Desde la perspectiva de la diversificación internacional de una empresa, a medida que ésta aumenta también lo hace la distancia cultural entre su país de origen y los países de sus clientes extranjeros (Tihanyi et al., 2005). En este sentido, la diversificación geográfica puede aumentar el riesgo de una empresa debido a la mayor complejidad y al aumento de incertidumbre relacionada con el funcionamiento en nuevos mercados, así como los costes en superar las barreras que supone este mayor distanciamiento. Las distancias geográficas y culturales han sido generalmente consideradas fuentes de riesgo o incertidumbre (Rothaermel et al., 2006). Las empresas generalmente tienen una comprensión más limitada de las instituciones, normas, preferencias del cliente en los mercados culturalmente distantes que, en aquellos más próximos, como resultado, tienen que incurrir en costos más altos para lidiar con la complejidad y la especificidad asociadas con el mercado culturalmente distante (Tihanyi et al., 2005). Estos autores estudian la relación de la distancia cultural con diversas variables en el contexto de la internacionalización, para 66 muestras independientes. En general, no encuentran evidencia estadística de relación entre distancia cultural y diversificación geográfica. Sin embargo, los resultados si son significativos en determinados sectores económicos y en estudios con muestras más recientes, sugiriendo para estos últimos que la diversificación geográfica cada vez se asocia más con un conjunto de países culturalmente diversos. 
En el estudio de Chao, Kim, Zhao, \& Hsu (2012) se analiza el efecto moderador de la distancia institucional entre la diversificación geográfica y el resultado de la empresa. Una de las medidas incluidas para medir esta variable es la distancia normativa entre ambos países, que la equiparan a la distancia cultural, la cual modera de forma significativa pero positivamente la relación entre diversificación internacional y el desempeño de la empresa, disminuyendo lentamente el impacto de la distancia cultural en los negocios internacionales. No obstante, los mismos autores sugieren que estos resultados son debidos a la composición de la muestra (Fortune's Global 500) y a la globalización, ya que para aquellas empresas que carecen de las habilidades de aprendizaje para convertir las diferencias culturales en innovaciones, la distancia cultural aún debería representar una amenaza.

A su vez, otras decisiones estratégicas de internacionalización también se ven afectadas por la DP, como es la adaptación de la estrategia de marketing, confirmándose su influencia positiva y significativa (Sousa y Lages, 2011; Navarro et al., 2014). Con respecto a la forma de entrada en mercados exteriores, investigaciones previas encuentran que la diversidad lingüística y la distancia cultural son indicadores estadísticamente significativos en su relación (Dow y Larimo, 2011), si bien otras investigaciones no encuentran evidencia empírica de relación con la distancia cultural (Rienda, Claver y Quer, 2013).

Por lo tanto, la mayoría de los argumentos previos tanto relacionados con la diversificación internacional como con otra decisión estratégica parecen apoyar la siguiente hipótesis:

- H1. La distancia psicológica (cultural, geográfica y lingüística) influye positivamente en la diversificación geográfica de una economía.

\subsection{Factores del entorno}

Cuando una empresa decide prolongar su actividad en mercados internacionales, el atractivo de un mercado exterior es uno de los principales aspectos a considerar en los factores de entorno, atractivo que se caracteriza por factores tales como el tamaño del mercado potencial y la alta estabilidad política y económica que favorecen un entorno empresarial propicio (Javalgi et al., 2003). Además, la intensidad competitiva de un país es otro de los elementos incluidos en el entorno externo (Navarro et al., 2014).

La estabilidad política y económica del país destino es un factor relevante en la decisión de las empresas en su proyección internacional (Javalgi y Grossman, 2014), estando la fortaleza económica de un país positivamente relacionada con el atractivo de un mercado exterior (Shankarmahesh et al., 2005). Los criterios económicos han sido ampliamente utilizados en los estudios de SME (Cavusgil et al., 2004; Rothaermel et al., 2006; Whitelock y Jobber 2004; Sheng y Mullen, 2011), así como el riesgo país ${ }^{9}$ de los mercados destino de las exportaciones o receptoras de la inversión (Rothaermel et al., 2006; Ojala y Tyrväinen, 2008; Slangen y Van Tulder, 2009; Rienda et al., 2013). Se sugiere que un alto nivel de PIB per cápita de un país es

9. En general, el riesgo país es la probabilidad de que se produzca una pérdida financiera por circunstancias macroeconómicas, políticas o sociales, o por desastres naturales, en un país determinado (Banco de España, 2008). 
un importante determinante en la decisión de entrar en un mercado exterior (Rothaermel et al., 2006), de la misma forma que, generalmente, las empresas evitan realizar IDE's en países con alto riesgo país (Quer et al., 2007; Rothaermel et al., 2006). A su vez, factores que condicionan la forma de IDE son el crecimiento del PIB del mercado extranjero (Quer, Claver y Rienda, 2017; Rienda et al., 2013; Dow y Larimo, 2011) y el riesgo asociado al país de destino (Rienda et al., 2013).

La intensidad competitiva de un país es otro de los factores del entorno incluido en el campo de la internacionalización, de forma que un elevado nivel de competencia influye positivamente en diferentes decisiones estratégicas en los mercados exteriores, tales como en aspectos organizacionales (Leonidou, 2000), en la SME (Whitelock y Jobber, 2004) y en el grado de adaptación del marketing mix (Powers y Loyka 2010; Navarro-García et al., 2014). Por su parte, para Shama (2000) el nivel de competencia es un factor a incluir en las estrategias de entrada de las empresas en mercados exteriores, aun siendo el mercado potencial el factor más determinante. En el trabajo de Ozturk et al., (2015), que proponen una herramienta para la SME, uno de los factores que también consideran determina la relativa conveniencia de escoger los mercados exteriores es la intensidad de la competencia.

Los factores del mercado son utilizados como factores explicativos y determinantes en la decisión de entrar en un mercado (Shama, 2000; Whitelock y Jobber, 2004; Sheng y Mullen, 2011; Sakarya et al., 2007; 0jala y Tyrväinen 2008; Gaston-Breton y Martin Martin, 2011). El tamaño del mercado y la tasa de crecimiento son los más utilizados para medir su potencial, según se desprende del estudio de Ozturk et al., (2015). En la literatura de la SME, el mercado potencial es un factor clave en la evaluación del mercado exterior (Robertson y Wood, 2001). Y, en el trabajo de Javalgi y Grossman (2014) se concluye que los factores relacionados con el mercado destino afectan positivamente en el grado de internacionalización, pero no así la estabilidad económica y política del país.

A falta de literatura previa referida a la diversificación geográfica, fundamentado en los anteriores argumentos que resaltan la importancia de los factores del entorno en la SME, y en estudios que los asocian con diversas decisiones estratégicas de internacionalización, se proponen las siguientes hipótesis:

- H2a. Los factores del entorno (desarrollo económico) influyen en la diversificación geográfica de una economía.

- H2b. Los factores del entorno (mercado) influyen en la diversificación geográfica de una economía.

\section{Las cooperativas agroalimentarias y la expansión internacional}

La importancia que adquiere la actividad internacional en las cooperativas agroalimentarias para su crecimiento (Bretos, Díaz-Foncea y Marcuello, 2018), hace que este trabajo sea de 
especial interés para este colectivo de empresas, sobre todo teniendo en cuenta que las limitaciones internas y externas de las cooperativas les restan competitividad y dificultan su expansión internacional.

La internacionalización se señala como uno de los elementos esenciales en el reto que supone para las cooperativas la globalización (Bretos y Marcuello, 2017). Asimismo, se identifica como uno de los seis factores clave en la competitividad de las cooperativas agroalimentarias españolas (Lajara-Camilleri y Server-Izquierdo, 2017), tema que ha suscitado considerable interés, tal como queda demostrado en trabajos previos (e.g. Medina, Mozas, Bernal y Moral, 2014; Fayos y Calderón, 2013; Juliá, Meliá y García, 2012; Fuentes et al., 2011). No obstante, su estudio está aún limitado (Bretos y Marcuello, 2017), orientándose las investigaciones existentes principalmente en aspectos organizacionales, económicos y comerciales (e.g. Juliá et al., 2012; Moyano, Puig y Bruque, 2008), así como en la productividad de las cooperativas (e.g. Martínez-Victoria, Maté Sánchez-Val y Arcas-Lario, 2018; Aldaz y Millán, 2003).

Los trabajos sobre la internacionalización de las cooperativas agroalimentarias españolas, se centran principalmente en determinar los factores que influyen en la exportación (e.g. Medina et al., 2014), en aportar información sobre el grado de internacionalización (e.g. Fuentes, Sánchez Cañizares y Santos, 2011) y en identificar los problemas en su desarrollo internacional (e.g. Fayos y Calderón, 2013). También se distinguen otros trabajos que tratan la internacionalización del sector cooperativo de forma indirecta, como en Triguero-Cano y Cuerva (2011), al incluir la vocación exportadora, como característica endógena de la empresa, en el análisis entre grado de innovación con resultado empresarial, o en Mozas, Bernal, Fernández, Medina y Puentes (2020), que se trata la variable grado de internacionalización en su relación con la innovación de la organización.

Las limitaciones en la internacionalización de las cooperativas se establecen en diversas investigaciones. En el trabajo de Bretos et al. (2018), donde se analizan las 300 mayores cooperativas del mundo, relacionado con las características organizacionales y económicas, se identifican con: una limitada creación y dimensión marginal del sector cooperativo en la mayoría de países y sectores de actividad; su reducido tamaño; dificultad para atraer y retener directivos valiosos; los mecanismos ineficientes de este tipo de empresas para la toma de decisiones; y las limitaciones financieras para abordar una elevada inversión, además de una mayor asunción de riesgos.

A su vez, Fayos y Calderón (2013), en un estudio exploratorio realizado sobre una muestra de cooperativas agroalimentarias con alto grado de internacionalización, encuentran que las principales barreras externas radican en la competencia y en la gran distribución, por el gran tamaño y la dificultad de negociar. Mientras que las barreras internas se centran en la escasez de recursos financieros y en la falta de imagen corporativa, de marca e innovación. En este sentido, Juliá et al. (2012) identifican la innovación, internacionalización y diversificación, como factores de competitividad de los principales grupos cooperativos agroalimentarios en la Unión Europea. De igual forma que Medina et al. (2014), sobre una muestra de cooperativas agroalimentarias con web propia, concluyen que el tamaño de la empresa, la formación académica del gerente y realizar ventas a través de internet, influyen positivamente en la actividad 
exportadora de estas empresas. Aspectos que, según estos autores, se deberían considerar para acceder a nuevos mercados tanto de economías desarrolladas como emergentes (China, Rusia o Brasil).

Si bien las cooperativas se enfrentan a problemas del entorno, internos y estratégicos que les restan competitividad, los resultados de Bretos et al. (2018) nos hacen ser positivos. Estos autores concluyen que las grandes cooperativas internacionalizadas compiten de manera eficaz en los principales sectores económicos y países, con un incremento mayor en términos de facturación y empleo frente a las que operan exclusivamente a nivel doméstico. Sobresale por encima del resto el sector 'Agricultura y alimentación', con el 97\% de las cooperativas internacionalizadas. Para las mismas, la exportación se configura como la principal forma de entrada en mercados exteriores frente al resto de modalidades (acuerdos contractuales: licencias, franquicias; acuerdos accionariales: joint-venture, filial propia ${ }^{10}$.

Los motivos de la prevalencia de la modalidad de exportación en el sector agroalimentario, viene explicado por Fernández-Olmos y Díez-Vial (2014). Entre ellos, se citan, el menor coste y mayor flexibilidad que las otras formas de entrada, los avances tecnológicos en la conservación de los alimentos para su transporte, y el incremento de la oferta de estrategias comerciales y de producción innovadoras, acompañado con una menor demanda interna. Así, en la literatura existe consenso de la relevancia del sector de actividad de la empresa para explicar las diferencias en los modos de entrada (Tihanyi et al., 2005).

\subsection{El sector cooperativo agroalimentario exportador de Cataluña}

A fin de contextualizar las cooperativas agroalimentarias catalanas exportadoras en el presente estudio, en este apartado se detallan sus características principales y en especial de aquellas pertenecientes a la provincia de Lleida.

Como información previa, destaca Cataluña al situarse entre las primeras cinco comunidades autónomas en cuanto al número de cooperativas y cifra de facturación en el total del cooperativismo agroalimentario español, con aproximadamente un $10 \%$ y un $7 \%$ respectivamente ${ }^{11}$. Por lo que respecta al sector agroalimentario español en su conjunto, Cataluña es la segunda comunidad autónoma exportadora, con una cuota de exportación ${ }^{12}$ del $21 \%$, únicamente superada por Andalucía, con un $23 \%{ }^{13}$.

10. Modos de entrada definidos por Brouthers y Hennart, 2007.

11. Informe del cooperativismo agroalimentario Macromagnitudes del Cooperativismo Agroalimentario Español (2017). Disponible en: http://www.agro-alimentarias.coop/cooperativismo_en_cifras (consultado septiembre 2019).

12. Porcentaje de exportaciones de cada comunidad autónoma sobre el total de exportaciones agroalimentarias españolas.

13. Departamento de Aduanas de la Agencia Tributaría de España (AEAT). Datos 2017. 
Fundamentado en el estudio de Cos et al. (2019b), de una muestra de 204 cooperativas agroalimentarias catalanas ${ }^{14}$ socias de la FCAC (Federación de Cooperativas Agrarias de Cataluña), un 36\% son exportadoras, generando el 70\% de la facturación global de las cooperativas, siendo, además, su facturación media cuatro veces superior a la media de las no exportadoras. De su actividad internacional se subraya que: Lleida tiene una exportación global superior a la suma de las otras tres provincias catalanas y la media de exportación anual más alta (3.739 miles de €); las cooperativas del sector hortofrutícola son las más internacionalizadas, con valores de sus indicadores muy superiores al resto de orientaciones productivas, siendo a su vez el sector que factura más; el sector del aceite de oliva ocupa la segunda posición respecto a la cifra global de exportación, aunque en el periodo analizado se ha producido una atomización del sector; $y$, las cooperativas medianas/grandes ${ }^{15}$ con transformación ${ }^{16}$ sobresalen respecto al resto de tamaños, concentrando el 80\% del total exportado por las cooperativas agroalimentarias catalanas.

Comparando los datos de las cooperativas de la muestra anterior con los datos de las exportaciones totales de Cataluña, obtenidos de la AEAT, se observa una exportación media muy similar, 2.726 miles de € y 3.611 miles de € (año 2016), respectivamente, con un crecimiento porcentual superior tanto en el número de cooperativas exportadoras como en sus exportaciones totales, entre 2006-2016.

Respecto a Lleida, tal como se menciona en Cos et al. (2019b), con el 53\% de la facturación total de las cooperativas estudiadas, se configura como la principal provincia catalana exportadora. Con la mitad de sus cooperativas exportadoras, éstas realizan casi el $60 \%{ }^{17}$ de las exportaciones del sector cooperativo agroalimentario de Cataluña. A su vez, siendo el sector hortofrutícola el más exportador, que representa el 55\% del total exportado por las cooperativas agroalimentarias catalanas, el 80\% corresponde a Lleida, importancia que viene determinada, asimismo, por el hecho de que el $40 \%$ de las exportaciones agroalimentarias totales de esta provincia son también hortofrutícolas ${ }^{18}$.

En esta línea, destacar que la práctica totalidad de las organizaciones de productores de frutas y hortalizas $(\mathrm{OPFH}){ }^{19}$ se concentran en la provincia de Lleida (Cos, Escardíbul y Gallego, 2021).

14. En este estudio se obtienen los principales indicadores de competitividad del cooperativismo agroalimentario en Cataluña (2006-2016), tras agregar y depurar los datos primarios facilitados por la FCAC.

15. Clasificación basada en la Recomendación 2003/361/EC, referente a la definición de micro, pequeñas y medianas empresas.

16. Realizan un proceso de transformación generando más valor añadido.

17. 115.918 miles de $€$.

18. Además, la fruta es el producto más exportado en el total de la provincia de Lleida (supone el 26,6\%).

19. Entre las que se incluyen las cooperativas. 
Tabla 2. Principales países de exportación de las cooperativas agroalimentarias catalanas, ordenados por \% facturación global del 2016

\begin{tabular}{|c|c|c|c|}
\hline País destino & 2016 & 2014 & 2011 \\
\hline Alemania & 29,6 & 14,1 & 20,1 \\
\hline Francia & 21,6 & 22,6 & 24,4 \\
\hline Italia & 6,5 & 5,6 & 10,1 \\
\hline Bélgica & 5,4 & 5,8 & 2,1 \\
\hline EEUU & 5,2 & 6,7 & 2,9 \\
\hline Portugal & 5,2 & 3,4 & 4,4 \\
\hline Reino Unido & 3,3 & 4,1 & 4,1 \\
\hline Polonia & 3,2 & 4,1 & 2,3 \\
\hline Países Bajos & 2,8 & 4,3 & 3,4 \\
\hline Bulgaria & 1,8 & 3,5 & - \\
\hline Brasil & 1,7 & 3,0 & 1,2 \\
\hline Rusia & 1,3 & 1,2 & 1,5 \\
\hline Japón & 0,9 & 1,4 & -- \\
\hline Israel & 0,8 & -- & - \\
\hline Suiza & 0,7 & 1.9 & - \\
\hline Grecia & 0,7 & -- & - \\
\hline Emiratos Árabes Unidos & 0,6 & 1,0 & - \\
\hline China & 0,6 & 0,9 & - \\
\hline Andorra & 0,5 & 1,0 & -- \\
\hline Dinamarca & 0,4 & 1,3 & 1,9 \\
\hline Argelia & 0,3 & -- & - \\
\hline
\end{tabular}

Fuente: Elaboración propia. Anuarios socioeconómicos de las cooperativas agrarias de Cataluña FCAC.

De los anuarios socioeconómicos ${ }^{20}$ de la FCAC, se obtienen los principales destinos de exportación de las cooperativas agroalimentarias de Cataluña. En la Tabla 2 se observa una tendencia a la diversificación del número de destinos, pasando de 12 a 22 países atendidos, entre 2011 y 2016. Sin embargo, el porcentaje de facturación de las cooperativas dedicado a países

20. El Observatorio Socioeconómico del Cooperativismo Agroalimentario Español (OSCAE) y la FCAC publican las principales macromagnitudes del cooperativismo agrario en España y en Cataluña respectivamente. El OSCAE (financiada por el Ministerio de Agricultura, Pesca y Alimentación (MAPA)) es la herramienta estadística de esta organización, que refleja las magnitudes más relevantes del cooperativismo español, a través de la encuesta anual «Directorio de Cooperativas». 
de la UE se ha visto incrementado entre 2011 (72\%) y 2016 (80,5\%), aunque en 2014 haya sufrido una disminución respecto al 2011, con un 68,8\% destinado a la UE. Estas cifras indican, por un lado, una mayor diversificación en el número de destinos, y, por otro lado, una mayor concentración del porcentaje de exportación a los países de la UE. De esta forma, en la selección de los destinos principales de exportación de las cooperativas, prima la pertenencia al Mercado Común Europeo y la proximidad geográfica, que simplifica el proceso exportador.

\section{Metodología}

\subsection{Muestra y variables}

Los datos del estudio son obtenidos de varias fuentes de información secundarias. La primera de ellas, procedente del Departamento de Aduanas de la Agencia Tributaria de España, se utiliza para obtener los países que componen la muestra, los destinos principales de las exportaciones agroalimentarias de las 672 empresas exportadoras de la provincia de Lleida ${ }^{21}$, que representan el 30\% del total de empresas exportadoras de Lleida. Aunque, si el estudio se centra en los exportadores regulares (empresas que han exportado en los cuatro últimos años consecutivos), el número de empresas agroalimentarias se reduce a la mitad (301), que suponen el $40 \%$ del total de empresas exportadoras de Lleida. La importancia que adquieren las cooperativas agroalimentarias de Lleida en estas cifras, aún representado el 10\%, hay que analizarla en el contexto del sector cooperativo agroalimentario de Cataluña, al ser la provincia más internacionalizada, tal como se ha mencionado en el apartado anterior de este artículo. A su vez, prevalece el sector hortofrutícola por encima del resto de sectores económicos, tanto para el total de las exportaciones agroalimentarias de Lleida como para el sector cooperativo de esta provincia.

El resto de las fuentes secundarias proporcionan la información relativa a las variables del estudio para cada país destino prioritario: Banco Mundial, World Development Indicators Database (WDI); OCDE; OMC; Global Competitiveness Report (World Economic Forum); Hofstede Centre (2010); Geobytes Database; y, Centre for the Practice of International Trade (Melbourne Business School). Tras eliminar aquellos países prioritarios sin datos disponibles en dos o más de las variables utilizadas, la muestra finalmente obtenida se compone de 23 países, que representan el 83,4\% de las exportaciones totales del sector y ámbito geográfico estudiado.

En la Tabla 3 se muestra la descripción de las variables. La diversificación geográfica, como variable dependiente, generalmente viene determinada por el número de países donde la empresa desarrolla su actividad. No obstante, en este trabajo, al realizarse el análisis desde la perspectiva generalista de una economía y atendiendo al carácter dinámico de esta estrategia, se fundamenta en la 'Tasa de crecimiento', durante un determinado periodo, de las exportaciones a cada destino seleccionado.

21. Fuente: Estacom del Instituto Español de Comercio Exterior (ICEX). 


\section{Tabla 3. Descripción de las variables}

\begin{tabular}{|c|c|c|}
\hline Variable & Unidad de medida & Descripción \\
\hline $\begin{array}{l}\text { Tasa de crecimiento } \\
\text { (TC) }\end{array}$ & Porcentaje & $\begin{array}{l}\text { Tasa de variación, de } 2010 \text { a 2014, de las exportacio- } \\
\text { nes de la economía analizada dirigidas a cada país } \\
\text { destino. }\end{array}$ \\
\hline $\begin{array}{l}\text { Distancia cultural } \\
\text { (DC) }\end{array}$ & $\begin{array}{l}\text { Índice de Kogut y Singh } \\
\text { (1988) }\end{array}$ & 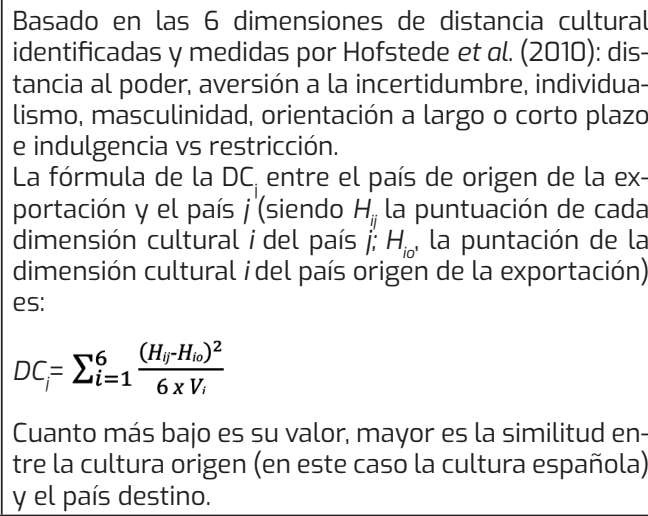 \\
\hline $\begin{array}{l}\text { Distancia geográfica } \\
\text { (DG) }\end{array}$ & Kilómetros & $\begin{array}{l}\text { Kilómetros entre el país de origen y cada país destino. } \\
\text { Se realiza una transformación logarítmica. }\end{array}$ \\
\hline $\begin{array}{l}\text { Diversidad } \\
\text { lingüística (DL) }\end{array}$ & $\begin{array}{l}\text { Factor de } 3 \text { items } \\
\left(L_{1}, L_{2}, L_{3}\right): \text { Lang }^{f}\end{array}$ & $\begin{array}{l}\text { Escala desarrollada por Dow y Karunaratna }(2006) \text {, } \\
\left.\text { que miden ésta variable como un factor (Lang }{ }^{f}\right) \text { de } \\
\text { tres ítems }(\mathrm{L} 1, \mathrm{~L} 2, \mathrm{~L} 3) \text {, los cuales reconocen la hetero- } \\
\text { geneidad de lenguas entre dos países. } \\
\text { Cada ítem se mide en una escala de cinco catego- } \\
\text { rías: el valor } 5 \text { corresponde a la máxima diversidad } \\
\text { lingüística entre las naciones origen y destino de la } \\
\text { exportación, mientras que el valor } 1 \text { representaría la } \\
\text { mínima diversidad. }\end{array}$ \\
\hline $\begin{array}{l}\text { Intensidad } \\
\text { económica }\end{array}$ & PIB per cápita & $\begin{array}{l}\text { Renta per cápita, medido en USD. Se realiza una trans- } \\
\text { formación logarítmica. }\end{array}$ \\
\hline Riego país & $\begin{array}{l}\text { Índice de riesgo país } \\
\text { CRAM }\end{array}$ & $\begin{array}{l}\text { Índice de riesgo país CRAM de la OCDE (2015), que } \\
\text { mide el riesgo en una escala del } 0 \text { al } 7 \text {. } \\
\text { Valor } 0 \text {, mínimo riesgo país; valor } 7 \text {, máximo riesgo. }\end{array}$ \\
\hline $\begin{array}{l}\text { Competitividad país } \\
\text { (GCl) }\end{array}$ & $\begin{array}{l}\text { Índice de Competitividad } \\
\text { Global }\end{array}$ & $\begin{array}{l}\text { Índice del World Economic Forum (2014-2015), con una } \\
\text { escala 1-7. } \\
\text { Cuanto más baja sea la puntuación del país menor } \\
\text { será su competitividad; el valor máximo es para Suiza } \\
\text { con 5,70 y el mínimo para Guinea, con un valor del } \\
\text { 2,79, para 2014-2015. }\end{array}$ \\
\hline Población & Miles habitantes & $\begin{array}{l}\text { Miles de habitantes. Se realiza una transformación } \\
\text { logarítmica. }\end{array}$ \\
\hline $\begin{array}{l}\text { Cuota de } \\
\text { importación }\end{array}$ & Porcentaje & $\begin{array}{l}\text { Cuota de importación mundial de cada país destino de } \\
\text { la economía analizada. }\end{array}$ \\
\hline
\end{tabular}

Fuente: Elaboración propia. 
En cuanto a las variables independientes, la primera de ellas la distancia psicológica, por su naturaleza multidimensional reflejado en estudios previos (Dow y Larimo, 2011; Sheng y Mullen, 2011; Malhotra, Sivakumar y Zhu, 2009), incluye la distancia cultural (DC), distancia geográfica (DG) y diversidad lingüística (DL). Para representar la DC, la medida más utilizada, en el ámbito de la internacionalización, es el índice compuesto de Kogut y Singh ${ }^{22}$ (1988) (e.g. Malhotra et al., 2009; Dow y Larimo, 2011), basado en las 6 dimensiones de la cultura nacional identificadas y medidas por Hofstede et al. (2010). Para la DG, siguiendo a otros trabajos (e.g. Buckley et al., 2007; 0jala y Tyrvainen, 2007), se mide la distancia en kilómetros entre dos países. Y, por lo que respecta a la DL, se ha adoptado la escala desarrollada por Dow y Karunaratna (2006), comúnmente utilizada en investigaciones anteriores (e.g. Dow y Larimo, 2011; Sheng y Mullen, 2011), que mide ésta variable como un factor ( Lang $^{f}$ ) de tres ítems $\left(L_{1}, L_{2}, L_{3}\right)$, los cuales reconocen la heterogeneidad de lenguas entre dos países.

Dentro de los factores del entorno, el desarrollo económico se mide a través de la intensidad económica, del riesgo país y de la intensidad competitiva de cada país estudiado. Para ello se utilizan, respectivamente, el PIB per cápita, obtenido de los Indicadores de Desarrollo Mundial (2014) del Banco Mundial, el Índice de riesgo CRAM de la OCDE (2015), que mide el riesgo en una escala del 0 al 7, y el Índice de Competitividad Global (GCI) del World Economic Forum (2014-2015), con una escala 1-7. Mientras la población y la cuota de importación mundial de cada país destino se han utilizado para medir el tamaño del mercado. Dicha información proviene del Banco Mundial y de la Organización Mundial de Comercio (OMC) (2013), respectivamente.

Además, se ha optado por la transformación logarítmica de varios de los indicadores anteriores (DG, PIB per cápita y Población) para normalizar su distribución (Rienda et al., 2013; Quer et al., 2017; Cos y Escardíbul, 2019).

En la Tabla 4 se detallan los países destino de la muestra, el valor de exportación, la tasa de crecimiento de las exportaciones en el periodo analizado y los valores de las variables utilizadas en el estudio.

La UE-28 concentra la mayoría de las ventas exteriores del sector descrito $(61,6 \%)$, si bien la mitad de las exportaciones agroalimentarias se dirigen a países de la Unión Europea de los quince (UE-15), destacando de forma notoria Francia que ocupa la primera posición, con un $17,1 \%$ de la cuota de exportación. Le siguen, en orden de importancia, Italia y Alemania, que junto a Francia representan el 38,9\% del total exportado por las empresas agroalimentarias de Lleida. La mayor facilidad de exportación a estos países, tanto por pertenecer a una zona aduanera común como por su proximidad geográfica, prima en su elección. Para el resto de áreas geográficas ${ }^{23}$, las exportaciones se encuentran repartidas entre el Resto de Europa (8\%), América del Norte (3,2\%), América Latina (5,3\%) y el Resto del mundo (principalmente Asia y África) que obtiene la máxima participación, con el 21,8\%.

22. Basado en el índice de Hofstede (1980).

23. Clasificación seguida por Dejo y Ramírez (2010). Los países se clasifican en áreas geográficas, siguiendo criterios económicos, sociales, políticos y/o geográficos, que permitan mantener la homogeneidad dentro de cada área establecida y la heterogeneidad entre ellos. 


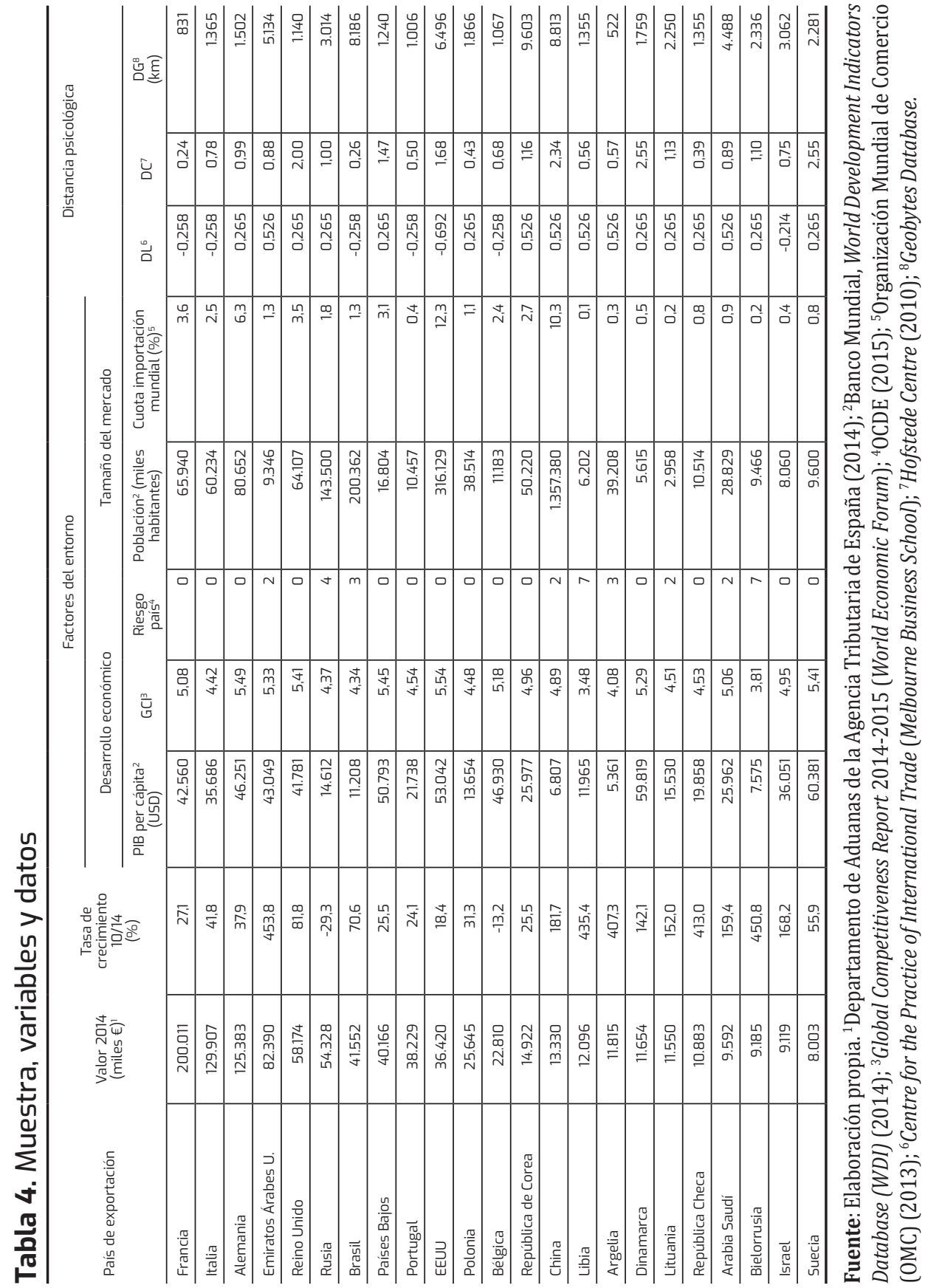


No obstante, el crecimiento de las exportaciones agroalimentarias de Lleida, en el periodo analizado, ha sido bastante inferior en los destinos principales de la UE-28 que en el resto de países con participaciones más marginales, adquiriendo estos últimos mayor protagonismo en detrimento a los destinos comunitarios ${ }^{24}$. Todo ello denota una tendencia a la diversificación de las exportaciones, al dirigirse a otras áreas del mundo más dinámicas y de mayor amplitud, tanto de economías avanzadas como emergentes, pese a seguir predominando una marcada concentración geográfica de las ventas exteriores de Lleida hacia países comunitarios y en especial a la UE-15.

Comparando estos datos con las exportaciones de las cooperativas agroalimentarias catalanas, el porcentaje de exportación dedicado a los países de la UE-28 (véase apartado 3.1) también disminuye entre 2011 y 2014, no obstante, en 2016 se produce un incremento, a diferencia del total de exportaciones agroalimentarias de Lleida, que la participación de las exportaciones dirigidas a la UE-28 sigue siendo menor que en 2010, según datos de la AEAT.

\subsection{Método}

Siguiendo otros estudios relacionados con el ámbito de la presente investigación, primero se realiza un análisis factorial (e.g. Whitelock y Jobber 2004; Sakarya et al., 2007; Sheng y Mullen 2011), con objeto de reducir la dimensionalidad de las variables originales incluidas en el estudio, utilizando el método de componentes principales (ACP) con rotación Varimax y normalización de Kaiser, que tiene la ventaja de mejorar la interpretación de los factores subyacentes obtenidos.

En segundo lugar, con las puntuaciones de los factores retenidos del ACP, se lleva a cabo un análisis de regresión múltiple (e.g. Triguero-Cano y Cuerva, 2011; Benito-Osorio et al., 2016), evitando el problema de la correlación entre las variables y la influencia de un gran número de ítems. Con este modelo de regresión se realiza una estimación del efecto que tienen los factores de la SME sobre la diversificación geográfica de una economía concreta ('Tasa de crecimiento' de las exportaciones), contrastando las tres hipótesis de trabajo definidas en el marco teórico:

- H1. La distancia psicológica (cultural, geográfica y lingüística) influye positivamente en la diversificación geográfica de una economía.

- H2a. Los factores del entorno (desarrollo económico) influyen en la diversificación geográfica de una economía.

- H2b. Los factores del entorno (mercado) influyen en la diversificación geográfica de una economía.

24. Las exportaciones a la UE-28 han pasado de representar el 66,5\% del flujo exportador agroalimentario de Lleida, en el año 2010, al 61,6\%, en el año 2014. 


\section{Resultados}

El resultado del análisis factorial ${ }^{25}$ de las ocho variables originales del modelo se presenta en la Figura 1. Previamente, la prueba de esfericidad de Bartlett ( $\mathrm{p}<0.001$ ) es significativa, lo que sugiere que los datos son adecuados para el análisis factorial. Del ACP se obtienen cuatro factores, que conjuntamente explican el $88,1 \%$ de la varianza, con las denominaciones 'Desarrollo económico' (DE), 'Tamaño del mercado' (TM), 'Distancia cultural/Diversidad Lingüística' (DC/ DL) y 'Distancia geográfica (DG), de acuerdo a las variables que representan (cargas factoriales) ${ }^{26}$.

\section{Figura 1. Path diagram del ACP: Cargas factoriales}

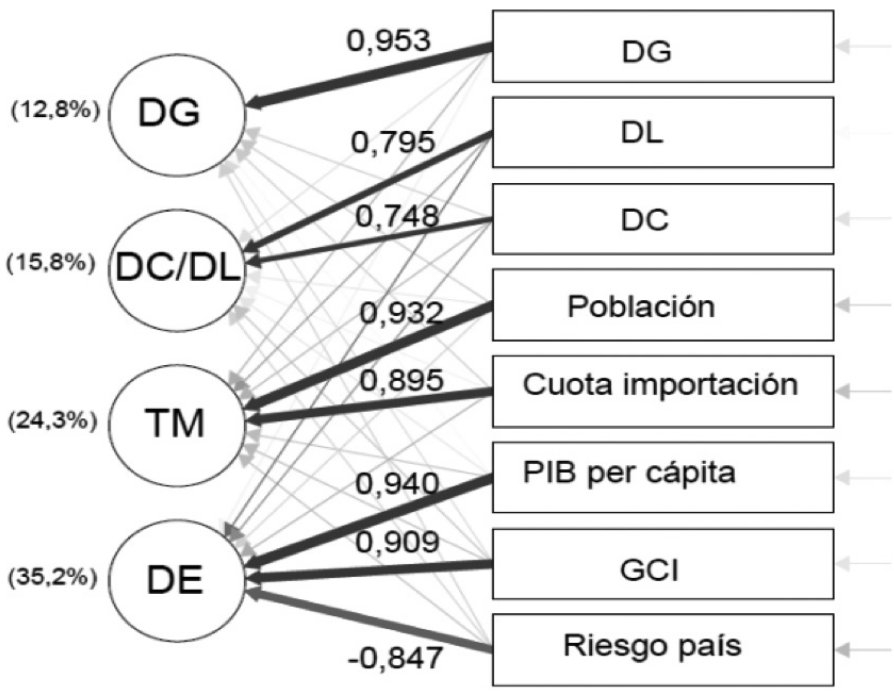

Notas: Método de extracción: Análisis de componentes principales; Método de rotación: Varimax con normalización de Kaiser; F1=Desarrollo económico (DE), F2=Tamaño del mercado (TM), F3=Distancia cultural/Diversidad (DC/DL) y F4=Distancia geográfica (DG); \% varianza explicada de cada factor entre paréntesis.

Fuente: Elaboración propia a partir de Cos y Escardíbul (2019).

25. Los análisis se han realizado con el software JASP (Versión 0.12). JASP Team (2020) [Computer software]. $<$ https://jasp-stats.org/download/>

26. Todas las correlaciones (cargas factoriales) son positivas, excepto para la variable 'Riesgo país' del primer factor, que es negativa. 
A continuación, se realiza un análisis de regresión siguiendo el método secuencial de selección backward (eliminación 'hacia atrás') (Tabla 5). La comparación del Modelo 1 con el Modelo 2 demuestra que incluir todas las variables independientes (Modelo 1) no aumenta su capacidad predictiva. En el Modelo 2, donde se eliminan las variables con una contribución significativamente menor, se incrementa el $\mathrm{R}^{2}$ ajustado y el valor de la $\mathrm{F}$, de mayor nivel de significación. Por tanto, se observa que la eliminación en el Modelo 1 de una variable mejora el análisis de regresión, siendo posiblemente la DG redundante. También se comprueba que ambos modelos están libres de autocorrelación, al ser el coeficiente de Durbin-Watson ${ }^{27}$ cercano a 2.

La especificación econométrica del modelo 2 estimado es la siguiente:

$$
\begin{gathered}
T C_{i}=146,130-87,078 * D E_{i}-53,640 * T M_{i}+40,022 * D C / D L_{i} \\
i=1 \ldots .23
\end{gathered}
$$

donde $\mathrm{TC}_{\mathrm{i}}$ es la tasa de crecimiento de exportación al país "i".

Se obtienen dos coeficientes de regresión predictivos significativos: el desarrollo económico $(\mathrm{p}=0,005)$ y el tamaño del mercado $(\mathrm{p}=0,06)$. Así, los resultados de este trabajo vienen a confirmar empíricamente la relación existente entre el desarrollo económico y el tamaño del mercado de un país extranjero con la variable dependiente, la tasa de crecimiento de las exportaciones de una economía. Además, no todas las variables independientes ejercen dicha influencia, como es el caso de la DC/DL, que, aunque con signo positivo en su coeficiente de regresión, éste no es significativo.

Los resultados del modelo planteado en la regresión permiten, pues, validar las H2a y H2b, en el sentido que el desarrollo económico (renta per cápita, intensidad competitiva, riesgo país) y el tamaño de mercado del país destino de las exportaciones influyen en la diversificación geográfica de una economía. No obstante, la H1, con los resultados obtenidos, no ha podido ser confirmada, al no encontrarse evidencia empírica de relación entre ninguna de las variables incluidas en la distancia psicológica (cultural/idiomática y geográfica) con la diversificación geográfica.

27. Durbin-Watson comprueba que las correlaciones entre los residuos se encuentran entre 1 y 3 , como se requiere, aunque lo ideal es próximo a 2. 
Tabla 5. Determinantes de la diversificación geográfica. Resultados de Regresión

\begin{tabular}{|c|c|c|}
\hline & Modelo 1 & Modelo 2 \\
\hline Variable (Factor) & Coeficiente b & Coeficiente b \\
\hline Desarrollo económico & $\begin{array}{c}-87,077^{\text {亦 }} \\
(27,778)\end{array}$ & $\begin{array}{c}-87,078^{* * *} \\
(27,048)\end{array}$ \\
\hline Tamaño del mercado & $\begin{array}{c}-53,640 \dagger \\
(27,777)\end{array}$ & $\begin{array}{c}-53,640 \dagger \\
(27,047)\end{array}$ \\
\hline $\mathrm{DC} / \mathrm{DL}$ & $\begin{array}{c}40,023 \\
(27,778)\end{array}$ & $\begin{array}{c}40,022 \\
(27,048)\end{array}$ \\
\hline DG & $\begin{array}{c}-3,368 \\
(27,773)\end{array}$ & \\
\hline Constante & 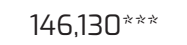 & 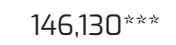 \\
\hline $\mathrm{R}^{2}$ & 0,465 & 0,465 \\
\hline Adjusted $\mathrm{R}^{2}$ & 0,346 & 0,380 \\
\hline F statistic & $3,911^{*}$ & $5,495^{\text {*स* }}$ \\
\hline Durbin-Watson statistic & 2,128 & 2,115 \\
\hline \multicolumn{3}{|l|}{ Variable dependiente } \\
\hline Tasa de crecimiento & & \\
\hline
\end{tabular}

Nota: Método de análisis Backward.

${ }^{* * *} \mathrm{p}<0,001,{ }^{* *} \mathrm{p}<0,01,{ }^{*} \mathrm{p}<0,05,{ }^{\dagger} \mathrm{p}<0,1$. Errores estándares entre paréntesis.

Fuente: Elaboración propia.

\section{Conclusiones}

La selección de mercados exteriores es una decisión estratégica clave y un factor decisivo de éxito en la estrategia de internacionalización de una empresa (He y Wei, 2011; Papadopoulos y Martín, 2011), avalado por la amplia literatura existente, si bien heterogénea y fragmentada. A pesar de su reconocida importancia en la literatura de los negocios internacionales, la mayoría de los trabajos sobre el proceso de internacionalización se analizan desde la decisión sobre la forma de entrada (Werner, 2002), siendo menores los estudios enfocados en la decisión de selección de mercados extranjeros (Andersen y Buvik, 2002). No obstante, se destaca una extensa literatura que detalla los criterios y modelos tanto teóricos como prácticos que pueden emplear las empresas en su decisión de seleccionar aquellos mercados más idóneos, y que no siempre siguen un proceso gradual. Además, existen muchas disparidades y poco consenso acerca del método ideal para su selección, a pesar de las similitudes en los diferentes modelos propuestos (Papadopoulos y Martín Martín 2011). Otros estudios tratan la relación de esta 
disciplina, la SME, con otras decisiones fundamentales de la internacionalización (e.g. Koch, 2001; Tihanyi et al., 2005; Navarro-García et al., 2014) y con el resultado de la empresa (e.g. Brouthers et al., 2009). En cuanto a la diversificación internacional, se trata de una estrategia ampliamente estudiada, reflejado en las múltiples investigaciones que la respaldan, aunque más enfocadas a multinacionales e IDE's, así como más vinculada a la influencia de los factores internos de la empresa, relegando aquellos de naturaleza externa.

En todo este contexto, la internacionalización, reconocida en la literatura como unos de los factores clave en la competitividad de las cooperativas agroalimentarias españolas, los estudios previos, siendo todavía limitados, se centran principalmente en aspectos más tradicionales, no encontrando dentro de la temática de este estudio, trabajos que lo aborden.

Con el presente trabajo se ha pretendido seguir avanzando en el conocimiento de la internacionalización de la empresa española, aportando una nueva evidencia empírica sobre un tema poco tratado, como es la relación entre la SME y la diversificación internacional. Tras la revisión de la literatura sobre la temática de estudio, se investiga y se comprueba que factores de la SME influyen en la diversificación geográfica, fundamentado en fuentes secundarias que nos proporcionan información objetiva y una accesibilidad inmediata. De esta forma, el estudio se ha centrado en el análisis conjunto de los factores del entorno y de la distancia psicológica, en el contexto de la tendencia a la diversificación de destinos de exportación de una economía concreta.

En el estudio de Cos y Escardibul (2019) se obtiene que la menor distancia psicológica percibida (principalmente la proximidad geográfica) es todavía un factor primordial en la selección de mercados extranjeros, pero la tendencia a diversificar los destinos de exportación en el periodo analizado, aunque con menores participaciones, va dando paso al empleo de otros factores usuales en el ámbito de la SME, como son el desarrollo económico y el tamaño de mercado. A su vez, según el trabajo, los países seleccionados, fruto de la diversificación geográfica, no están precisamente categorizados como mercados 'seguros', sino que suponen, la mayoría de ellos, destinos de riesgo e incertidumbre. Con la presente investigación se ha podido comprobar si la relación entre estos factores con la diversificación geográfica recibe apoyo empírico, planteando una serie de hipótesis, que han permitido dar respuesta a las preguntas planteadas, a partir de los resultados obtenidos.

Partiendo de un análisis factorial del que obtenemos cuatro factores, los resultados del análisis de regresión demuestran que el desarrollo económico y el tamaño del mercado se configuran como variables influyentes en la diversificación geográfica de una economía. Se puede observar, pues, que el modelo muestra una relación significativa y negativa entre el desarrollo económico y el tamaño de mercado de un país de exportación con la tendencia de una economía a la diversificación geográfica. Es decir, las empresas tienden a diversificar sus ventas hacia países con menor estabilidad política y económica y de menor tamaño de mercado. Se ha de considerar que el menor desarrollo económico de un país se traduce, en este estudio, en una renta per cápita más baja, una intensidad competitiva menor y en la existencia de riesgo país, que se identifica con inestabilidad política y económica del país destino. Otros trabajos se suman a estos resultados, si bien no son del todo comparables al tratarse de otras 
decisiones estratégicas diferentes a la tratada, pero que, sin embargo, sirven para comprobar la importancia en el área de la internacionalización. En estos estudios se concluye una relación entre factores del mercado destino y grado de internacionalización de una empresa (Javalgi y Grossman, 2014), entre intensidad competitiva y estrategias de marketing (Powers y Loyka 2010; Navarro-García et al., 2014) y entre indicadores del PIB (Rothaermel et al., 2006; Dow y Larimo, 2011: Quer et al., 2017), de riesgo país (Rothaermel et al., 2006; Rienda et al., 2013) y de nivel de competencia (Shama, 2000) con la forma de entrada.

Los resultados, no obstante, no proporcionan apoyo empírico para la distancia psicológica, no afectando este factor en ninguna de las vertientes analizadas (geográfica, cultural o idiomática), en la tendencia de una economía a diversificar los destinos de exportación. Investigaciones previas sobre la influencia de la DP muestran resultados contradictorios, ya que mientras Tihanyi et al. (2005) no pueden confirmar una relación entre distancia cultural y diversificación geográfica, excepto para determinados supuestos, Chao et al. (2012) si encuentran evidencia empírica de relación entre ambas variables, concluyendo que la distancia cultural cada vez afecta menos en los negocios internacionales. En el caso de la influencia de la distancia psicológica en otras decisiones estratégicas, su relación es positiva con la adaptación del marketing (Sousa y Lages, 2011; Navarro et al., 2014) y con la forma de entrada (Dow y Larimo, 2011), si bien para esta última decisión estratégica algunos estudios no encuentran asociación (Rienda et al., 2013).

Como parte de la economía analizada, las cooperativas agroalimentarias descritas en este artículo podrían utilizar estas conclusiones como referencia y comparativa de las empresas de la economía estudiada, considerando sus características concretas. La importancia de la internacionalización de las cooperativas para su competitividad ha quedado demostrada y la selección de mercados exteriores es una de las decisiones clave para la empresa en este proceso. La influencia de los criterios relevantes de la SME con diversas decisiones estratégicas de internacionalización, como es la diversificación geográfica, ha de servir a las cooperativas del presente estudio para poder evaluar las diferentes alternativas que se le presentan.

Del presente estudio se pueden arrojar una serie de implicaciones prácticas en el ámbito estudiado, que afectarían principalmente a las empresas exportadores de la economía analizada, incluyendo y por tanto afectando también a las cooperativas exportadoras del sector analizado, el agroalimentario. No obstante, por el carácter generalista de las variables utilizadas podría extenderse a otros territorios y/o sectores económicos. Diversificar destinos debería servir a las empresas para concienciarse acerca de la importancia de la selección de mercados en su proceso de internacionalización, aplicando, para ello, una metodología de enfoque sistemático o no, que les permita ampliar su abanico de mercados objetivo. Sobre todo, considerando que las empresas de su territorio y sector, sean competidoras o no, buscan nuevas opciones para dirigir sus ventas internacionales, más allá de la seguridad de mercados comunitarios, próximos geográficamente y favorecidos por las mayores facilidades en todo el entramado logístico y por el mercado común. Probablemente estas empresas que se dirigen a mercados no deseables a priori por sus características macroeconómicas, los seleccionan por una serie de motivos que, aunque desconocidos en este estudio, les ofrece alguna ventaja competitiva 
que los hace idóneos. Tal como se apunta en Navarro et al. (2014), si se desarrollan métodos de análisis del entorno y decisiones estratégicas de marketing adecuadas, la incertidumbre y la inestabilidad de los países extranjeros no tienen por qué tener efectos negativos en el resultado exportador. Asimismo, aunque un mercado culturalmente distante podría asociarse con grandes incertidumbres y costos, representa grandes oportunidades y podría ser una fuente de tecnología y conocimiento nuevos y avanzados (He y Wei, 2011).

Para al sector cooperativista agroalimentario la utilidad de estas implicaciones prácticas adquiere especial relevancia, al operar, la mayoría de las grandes cooperativas mundiales del sector agroalimentario, a nivel internacional (Bretos et al., 2018), destacándose, a su vez, la exportación como forma de entrada frente al resto de modalidades. Con estas premisas, el sector cooperativo estudiado aún tiene recorrido en su internacionalización, ya que la mitad de las cooperativas agroalimentarias de Lleida todavía no exportan. Además, con una tendencia a la concentración de sus exportaciones a países de la UE en cuanto a volumen se refiere, podrían fundamentarse en los factores del entorno para diversificar sus destinos, incrementando sus exportaciones en aquellos mercados ya atendidos, con aún participaciones marginales. Así, la proximidad geográfica y la pertenencia a la UE son los criterios principalmente utilizados por las cooperativas estudiadas para seleccionar mercados extranjeros, propio del enfoque no sistemático, lo que implica que para elegir países de exportación de otras economías avanzadas o emergentes deberían desarrollar modelos sistemáticos, que dotarían a las cooperativas de un método estructurado en la SME, con los beneficios que ello supone.

Si bien el presente estudio ha contribuido a progresar en el conocimiento de un ámbito temático concreto de la internacionalización empresarial y de una economía en particular, presenta una serie de limitaciones, que no influyen de forma significativa en los resultados y que podrían abrir camino a futuras investigaciones. Primero, la utilización exclusiva de fuentes de información secundarias de fácil acceso y objetivas, no permite conocer las opiniones del proceso de SME seguido por las empresas estudiadas. Segundo, la variable dependiente utilizada sirve para medir la tendencia a la diversificación geográfica de una economía determinada, pero los criterios de la literatura tradicional para medirla difieren si el análisis es a nivel empresa. Se propone, pues, una investigación que se fundamente en fuentes primarias para complementar y contrastar estos resultados. Por la relevancia del cooperativismo agroalimentario exportador, se podría plantear que el estudio fuese comparativo entre empresas cooperativas y no cooperativas. Y para finalizar, también se proponen investigaciones encaminadas a estudiar el efecto que la pandemia, Covid-19, ha tenido sobre las ventas exteriores de una economía, con objeto de determinar si se ha producido un desplazamiento significativo de los destinos seleccionados y en su caso averiguar los factores que han influido en su elección. 


\section{Bibliografía}

ALDAZ, N. \& MILLÁN, J. (2003): "Regional productivity of Spanish agriculture in a panel DEA framework", Appl. Econ. Lett, 10(2), 87-90.

AGUILERA-CARACUEL, J., DELGADO-MÁRQUEZ, B. \& VIDAL-SALAZAR, M.D. (2014): “Influencia de la internacionalización en el desempeño social de las empresas", Cuadernos de Gestión, 14(2), 15-3. DOI: 10.5295/cdg.130420ja.

ALBAUM, G., STRANDSKOV, J. \& DUERR, E. (2002): International Marketing and Export Management, Wokingham, UK: Addison-Wesley.

ALONSO, J.A. \& DONOSO, V. (1998): Competir en el exterior: la empresa española y los mercados internacionales, Madrid, Instituto Español de Comercio Exterior.

ANDERSEN, 0. \& BUVIK, A. (2002): "Firms' internationalization and alternative approaches to the international customer/market selection", International Business Review, 11, 347-363.

AYAL, I. \& ZIF, J. (1979): "Market expansion strategies in multinational marketing", The Journal of Marketing, 43(2), 84-94.

BANCO MUNDIAL (2015): World development indicators 2014. Disponible en: http://datos. bancomundial.org/indicador (Acceso Abril 2015).

BENITO-OSORIO, D., COLINO, A., GUERRAS-MARTÍN, LA. \& ZÚÑIGA-VICENTE, J.A. (2016): "The international diversification-performance link in Spain: Does firm size really matter?", International Business Review, 25(2), 548-558. D0I: 10.1016/j.ibusrev.2015.09.004.

BOATENG, A., QIAN, W. \& TIANLE, Y. (2008): "Cross-border M\&As by Chinese firms: An analysis of strategic motives and performance", Thunderbird International Business Review, 50, 259270 .

BRETOS, I. \& MARCUELLO, C. (2017): "Revisiting globalization challenges and opportunities in the development of cooperatives", Annals of Public and Cooperative Economics, 88(1), 47-73.

BRETOS, I., DÍAZ-FONCEA, M. \& MARCUELLO, C. (2018): “Cooperativas e internacionalización: un análisis de las 300 mayores cooperativas del mundo", CIRIEC-España, Revista de Economía Pública, Social y Cooperativa, 92, 5-37.

BROUTHERS, L.E. \& NAKOS, G. (2005): "The role of systematic international market selection on small firms' export performance", Journal of Small Business Management, 43(4): 363-381.

BROUTHERS, K.D. \& HENNART, J.F. (2007): “Boundaries of the Firm: Insights from International Entry Mode Research", Journal of Management, 33(3), 395-425. 
BROUTHERS, L.E., MUKHOPADHYAY, S., WILKINSON, T.J. \& BROUTHERS, K.D. (2009): "International market selection and subsidiary performance: a neural network approach", Journal of World Business, 44 (3), 262-273.

CAVUSGIL, S.T. \& ZOU, S. (1994): "Marketing strategy-performance relationship: an investigation of the empirical link in export market ventures", Journal of Marketing, 58(1): 1-21.

BUCKLEY, P.J., CLEGG, J., CROSS, A., LIU, X., VOSS, H. \& ZHENG, P. (2007): "The determinants of Chinese outward foreign direct investment", Journal of International Business Studies, 38, 499518.

CAVUSGIL, S.T., KIYAK, T. \& YENIYURT, S. (2004): “Complementary approaches to preliminary foreign market assessment: country clustering and country ranking", Industrial Marketing Management, 33 (7), 607-617.

CENTRE FOR THE PRACTICE OF INTERNATIONAL TRADE (2015): Melbourne Business School: Measures of Psychic Distance Stimuli 2010. Disponible en:

https://sites.google.com/site/ddowresearch/home (Acceso Abril 2015).

COS, P., ALDAZ, N., ESCARDÍBUL, B., MILLÁN, J. \& MOLTÓ, M. (2019): Indicadors de competitivitat al cooperativisme agroalimentari a Catalunya (2006-2016), Barcelona, Universitat Oberta de Catalunya (UOC), Colección SEHEN.

COS, P., COLOM, A. \& CABASÉS, A. (2019): "Geographic export diversification: Determinants and their link with export performance", Thunderbird International Business Review, 61, 397411. DOI: $10.1002 /$ tie.21991.

COS, P. \& ESCARDÍBUL, B. (2019): "Clústers de países prioritarios para la exportación”, Cuadernos de Gestión, 19(1), 147-174. D0I: 10.5295/cdg.170733pc.

COS, P., ESCARDÍBUL, B. \& GALLEGO, J.M. (2021): Las OPFH y el comercio exterior de fruta en Cataluña. El caso de las frutas de hueso: melocotón, nectarina y paraguayo (Capítulo 8). En C0LOM, A. \& FLORENSA, R. (Dir.): Cooperativismo agroalimentario y las OPFH. Una panorámica mundial y la realidad actual europea y española, Navarra, Thomson Reuters Aranzadi, 263-294.

CHAO, M.C.-H., KIM, S.H., ZHAO, H. \& HSU, C.-C. (2012): "Performance Implications of MNEs' Diversification Strategies and Institutional Distance", Thunderbird International Business Review, 54, 667-681.

DEPARTAMENTO DE ADUANAS DE LA AGENCIA TRIBUTARIA DE ESPAÑA (2015): Datos estadísticos de Comercio Exterior de España. Disponible en: http://datacomex.comercio.es y http://estacom.icex.es (Acceso Abril 2015).

DOW, D. (2000): "A note on psychic distance and export market selection", Journal of International Marketing, 8 (1), 51-64. 
DOW, D. \& KARUNARATNA, A. (2006): “Developing a Multidimensional Instrument to Measure Psychic Distance Stimuli", Journal of International Business Studies, 37, 578-602.

DOW, D. \& LARIM0, J. (2011): "Disentangling the Roles of International Experience and Distance in Establishment Mode Choice", Management International Review, 513, 321-356.

FAYOS GARDÓ, T. \& CALDERÓN GARCÍA, H. (2013): "Principales problemas de internacionalización de las cooperativas agroalimentarias españolas", REVESCO, Revista De Estudios Cooperativos, 111, 32-59. DOI:10.5209/rev_REVE.2013.v111.42675.

FERNÁNDEZ-OLMOS, M. \& DÍEZ-VIAL, I. (2013): “The direct or indirect exporting decision in agri-food firms", Agribusiness, 30(2), 148-164.

FUENTES GARCÍA, F.J., SÁNCHEZ CAÑIZARES, S.M. \& SANTOS ROLDÁN, L.M. (2011): “Cooperativas agroalimentarias y exportación. El proceso de internacionalización de la Cooperativa del Valle de Los Pedroches (COVAP)", REVESCO, Revista De Estudios Cooperativos, 104, 38-62. DOI: 10.5209/rev_REVE.2011.v104.2.

GASTÓN-BRETÓN, C. \& MARTÍN MARTÍN, O. (2011): “International Market Selection and Segmentation: A Two-Stage Model”, International Marketing Review, 28 (3), 267-290.

Geobytes Database. Disponible en: http://geobytes.com/citydistancetool/ (Acceso Abril 2015).

HE, X., LIN, Z. \& WEI, Y. (2016): "International market selection and export performance: a transaction cost analysis", European Journal of Marketing, 50 (5/6), 916-941.

HE, X. \& WEI, Y. (2011): "Linking market orientation to international market selection and international performance?", International Business Review, 20(5), 535-546.

HITT, M.A., TIHANYI, L., MILLER, T. \& CONNELLY, B. (2006): "International diversification: Antecedents, outcomes, and moderators", Journal of Management, 32: 831-867.

HOFSTEDE, G. (1980): Culture's Consequences: International Differences in Work-related Values. Beverly Hills: Sage Publications.

HOFSTEDE CENTRE (2010): Dimension Data Matrix. Disponible en:

http://geerthofstede.eu/dimension-data-matrix y

https://geert-hofstede.com/national-culture.html (Acceso Abril 2015).

HOFSTEDE, G., HOFSTEDE, G.J. \& MINKOV, M. (2010): Cultures and Organizations. London: McGrawHill.

JAVALGI, R., GRIFFITH, D. \& WHITE, S. (2003): "An empirical examination of factors influencing the internationalization of service firms", Journal of Services Marketing, 17(2/3), 185-199. 
JAVALGI, R.G. \& GROSSMAN, D.A. (2014): "Firm Resources and Host-Country Factors Impacting Internationalization of Knowledge-Intensive Service Firms", Thunderbird International Business Review, 56, 285-300. D0I:10.1002/tie.21622.

JOHANSON, J. \& VAHLNE, J.E. (1990): “The mechanism of internationalization”, International Marketing Review, 7 (4), 11-24.

JOHANSON, J. \& VAHLNE, J.E. (2009): "The Uppsala internationalization process model revisited: from liability of foreignness to liability of outsidership", Journal of International Business Studies, 40(9), 1411-1431.

JULIÁ J.F., MELIÁ E. \& GARCÍA G. (2012): “Strategies developed by leading EU agrifood cooperatives in their growth model", Service Business, 6(1), 27-46.

KATSIKEA, E.S., THEODOSIOU, M., MORGAN, R.E. \& PAPAVASSILIOU, N. (2005): “Export market expansion strategies of direct-selling small and medium-sized firms: implications for export sales management activities", Journal of International Marketing, 13(2): 57-92.

KOCH, A.J. (2001): "Selecting overseas markets and entry modes: two decision processes or one?", Marketing Intelligence \& Planning, 19 (1), 65-75.

KOGUT, B. \& SINGH, H. (1988): "The Effect of Nacional Cultura on the Choice of Entry Mode", Journal of International Business Studies, 19, 411-432.

LAJARA-CAMILLERI, N. \& SERVER-IZQUIERDO, R. (2017): “CCómo se puede mejorar la competitividad de las cooperativas agroalimentarias?", CIRIEC-España, Revista de Economía Pública, Social y Cooperativa, 90, 103-121.

LEE, C.S. \& YANG, S. (1990): "Impact of export market expansion strategy on export performance", International Marketing Review, 7(4): 41-51.

LEONIDOU, L.C. (2000): "Barriers to export management: an organizational and internationalization analysis", Journal of International Management, 62, 121-148.

LOSADA, F., RUZO, E., BARREIRO, J.M. \& NAVARRO, A. (2007): "Influencia de la estrategia de expansión internacional y de las características empresariales sobre el resultado exportado", Tribuna de Economía ICE, 837, 255-272.

MALHOTRA, S., SIVAKUMAR, K. \& ZHU, P.C. (2009): "Distance factors and target market selection: the moderating effect of market potential", International Marketing Review, 26 (6), 651673.

MARTÍNEZ-VICTORIA, M., MATÉ SÁNCHEZ-VAL, M. \& ARCAS-LARIO, N. (2018): "Spatial determinants of productivity growth on agri-food Spanish firms: a comparison between cooperatives and investor-owned firms", Agricultural Economics, 49, 213-223. D0I:10.1111/agec.12410. 
MAS, F.J., NICOLAU, J. \& RUIZ, F. (2006): "Foreign diversification vs concentration strategies and firm performance: moderating effects of the market, product and firm factors", International Marketing Review, 23(1): 54-82.

MEDINA, M.J., MOZAS, A., BERNAL, E. \& MORAL, E. (2014): "Factores determinantes para la exportación en las empresas cooperativas oleícolas andaluzas", CIRIEC-España, Revista de Economía Pública, Social y Cooperativa, 81, 241-262.

MOYANO, J., PUIG, F. \& BRUQUE, S. (2008): "Los determinantes de la competitividad en cooperativas", CIRIEC-España, Revista de Economía Pública, Social y Cooperativa, 61, 233-249.

MOZAS, A., BERNAL, E., FERNÁNDEZ, D., MEDINA, M.J. \& PUENTES, R. (2020): “Cooperativismo de segundo grado y adopción de las TIC", CIRIEC-España, Revista de Economía Pública, Social y Cooperativa, 100, 67-85. DOI: 10.7203/CIRIEC-E.100.17712.

NAVARRO, A. (2002): "Efecto de la estrategia de expansión exterior sobre el resultado exportador de la organización", Información Comercial Española, 802: 99-115.

NAVARRO-GARCÍA, A., ARENAS-GAITÁN, J. \& RONDÁN-CATALUÑA, F.J. (2014): “External environment and the moderating role of export market orientation", Journal of Business Research, 67(5), 740-745.

OJALA, A. \& TYRVAINEN, P. (2007): "Market entry and priority of small and medium-sized enterprises in the software industry: an empirical analysis of cultural distance. geographic distance and size", Journal of International Marketing, 15 (3), 123-149.

OJALA, A. \& TYRVÄINEN, P. (2008): "Market entry decisions of US small and medium-sized software firms", Management Decision, 46 (2), 187-200.

ORGANIZACIÓN MUNDIAL DEL COMERCIO (OMC) (2015): Estadísticas de Comercio internacional 2013, disponible en: https://www.wto.org (Acceso Abril, 2015).

ORGANIZACIÓN PARA LA COOPERACIÓN Y DESARROLLO ECONÓMICO (OCDE) (2015): Country Risk Assessment Model CRAM. Disponible en: http://www.oecd.org (Acceso Abril 2015).

OZTURK, A., JOINER, E. \& CAVUSGIL, S.T. (2015): “Delineating Foreign Market Potential: A Tool for International Market Selection", Thunderbird International Business Review, 57, 119-141. DOI:10.1002/tie.21686.

PAPADOPOULOS, N., CHEN, H. \& THOMAS, D.R. (2002): "Toward a tradeoff model for international market selection", International Business Review, 11 (2), 165-192.

PAPADOPOULOS, N. \& DENIS, J.E. (1988): "Inventory, taxonomy and assessment of methods for international market selection”, International Marketing Review, 5 (3), 38-51. 
PAPADOPOULOS, N. \& MARTÍN MARTÍN, O. (2011): "International market selection and segmentation: perspectives and challenges", International Marketing Review, 28 (2), 132-149.

POWERS, T.L. \& LOYKA, J.J. (2010): "Adaptation of marketing mix elements in international markets", Journal of Global Marketing, 23 (1), 65-79.

QUER, D., CLAVER, E. \& RIENDA, L. (2007): "The impact of country risk and cultural distance on entry mode choice: an integrated approach", Cross Cultural Management: An International Journal, 14(1), 74-87.

QUER, D., CLAVER, E. \& RIENDA, L. (2017): “Chinese multinationals in Spain: Determinants of establishment mode choice", Cuadernos de Gestión, 17(2), 15-36. D0I: 10.5295/cdg.150548dq.

RIENDA, L., CLAVER, E. \& QUER, D. (2013): “Formas de establecimiento en el mercado extranjero: factores determinantes para el caso de la multinacional india Tata", Cuadernos de Gestión, 13(2) (Año), 89-109. DOI: 10.5295/cdg.120335lr.

ROTHAERMEL, F., KOTHA, S. \& STEENSMA, K. (2006): "International market entry by US internet firms: an empirical analysis of country risk, national culture, and market size", Journal of Management, 32 (1), 56-82.

ROBERTSON, K.R. \& WOOD, V.R. (2001): "The Relative Importance of Type of Information in the Foreign Market Selection Process", International Business Review, 10(3), 363-79.

SAKARYA, S., ECKMAN, M. \& HYLLEGARD, K. (2007): "Market selection for international expansion; assessing opportunities in emerging markets", International Marketing Review, 24 (2), 208-238.

SHAMA, A. (2000): "Determinants of entry strategies of US companies into Russia, the Czech Republic, Hungary, Poland, and Romania", Thunderbird International Business Review, 42(6), 651-676.

SHANKARMAHESH, M.N., OLSEN, H.W. \& HONEYCUTT, E.D. (2005): "A dominant product-dominant country framework of industrial export segmentation", Industrial Marketing Management, 34 (3), 203-10.

SHENG, S.Y. \& MULLEN, M.R. (2011): "A hybrid model for export market opportunity analysis", International Marketing Review, 28 (2), 163-182.

SLANGEN, A. \& VAN TULDER, R.J. (2009): “Cultural distance, political risk, or governance quality? Towards a more accurate conceptualization and measurement of external uncertainty in foreign entry mode research", International Business Review, 183, 276-291.

SOUSA, C. \& LAGES, L.F. (2011): "The PD scale: a measure of psychic distance and its impact on international marketing strategy", International Marketing Review, 28 (2), 201-222. 
TRIGUERO-CANO, A. \& CUERVA, M.C. (2011): “El cooperativismo agroalimentario ante el reto de la globalización: renovarse o morir", CIRIEC-España, Revista de Economía Pública, Social y Cooperativa, 72, 97-124.

TIHANYI, L., GRIFFITH, D.A. \& RUSSELL, C.J. (2005): "The effect of cultural distance on entry mode choice, international diversification, and MNE performance: a meta-analysis", Journal of International Business Studies, 36(3), 270-283.

WERNER, S. (2002): "Recent developments in international management research: A review of 20 top management journals", Journal of Management, 28 (3), 277-305.

WHITELOCK, J. \& JOBBER, D. (2004): "An evaluation of external factors in decision of UK industrial firms to enter a new non-domestic market: an exploratory study", European Journal of Marketing, 38 (11/12), 1437-1455.

WORLD ECONOMIC FORUM (2015): The Global Competitiveness Report 2014-2015, Suiza. 